\title{
Patienten im Wettbewerb
}

\section{Erfahrungen als Landes-Patientenbeauftragte in Berlin}

\author{
Karin Stötzner
}

Karin Stötzner ist seit fast fünf Jahren nebenamtlich bei der Senatorin für Gesundheit, Umwelt und

Verbraucherschutz als

Patientenbeauftragte für das Land Berlin tätig. Hauptberuflich leitet die

Soziologin die Selbsthilfekontakt- und Informationsstelle SEKIS.

E-Mail

patientenbeauftragte@senguv.berlin.de
Kranke, behinderte und pflegebedürftige Menschen sind in einem zunehmend als Markt organisierten Gesundheitswesen auf wirksame individuelle und kollektive Verbraucherrechte angewiesen. Zur Stärkung der Souveränität von Patienten müssen insbesondere stimmige Konzepte für Beratung, Beteiligung und Empowerment entwickelt werden.

Patienten, Verbraucher, Kunden - Begrif$\mathrm{fe}$, die viele Patientinnen und Patienten nicht gerne in eine Reihe stellen. Das Selbstverständnis und die Anforderungen von und an Patienten wandeln sich jedoch so rasant, dass es sinnvoll ist, das Thema Patientenrechte auch unter dem Gesichtspunkt der Verbraucherpolitik zu betrachten.

Der zunehmende Wettbewerb im Gesundheitswesen, die Liberalisierung beispielsweise beim Vertrieb von Arzneimitteln, die Notwendigkeit, sich zwischen Behandlungs- und Diagnosemöglichkeiten selbst entscheiden zu müssen (aber auch zu können), die steigende Vielfalt von Tarifangeboten, Wahlmöglichkeiten und Eigenbeteiligungen bei und zwischen Krankenversicherungen stellen Patientinnen und Patienten vor neue Herausforderungen. Der Wunsch nach Selbstbestimmung und Souveränität bei der eigenen Gesundheit korrespondiert mit der Notwendigkeit zur Eigenverantwortung und Wahlnotwendigkeiten wie im Warenmarkt. Patientinnen und Patienten wollen und müssen sich im Feld der gesundheitlichen Versorgung in einem Markt, der einer der größten und am schnellsten wachsenden Märkte in Deutschland ist, bewegen und behaupten lernen.

$\mathrm{Zu}$ den wichtigsten Themen, die es für Patientinnen und Patienten als potenzielle Kunden zu diskutieren gilt, gehören Hilfen, Voraussetzungen und Bedingungen für Entscheidungen bei der Wahl
- von Behandlungs- und Diagnoseleistungen sowohl in der ambulanten wie in der stationären Versorgung: Hier geht es beispielsweise um die »Individuellen Gesundheitsleistungen « (Wahlleistungen in der Arztpraxis), Zahnersatz, wählbare Eingriffe der Krankenhausbehandlung.

- von Krankenkassen und Versicherungsleistungen

- beim Kauf von Medikamenten (Versandhandel und Internet, Möglichkeit zu Preisverhandlungen in der Apotheke).

Fachpolitische Forderungen werden daher vor allem erhoben zur

- Beseitigung von Intransparenz bezüglich sinnvoller (also wirksamer) und notwendiger Leistungen, vor allem aber bezüglich der Qualität erbrachter Gesundheitsleistungen

- Ausweitung an Information, Beratung und Aufklärung sowie

- Klärung und Konkretisierung von Rechten der Patienten im Versorgungsgeschehen.

Solche Feststellungen und entsprechende Forderungen haben beispielsweise der Sachverständigenrat zur Begutachtung der Entwicklung im Gesundheitswesen, Patientenberatungsstellen und die Verbraucherzentralen. Sie sind auch notwendige Konsequenz aus der Arbeit der Patientenbeauftragten für das Land Berlin, der einzigen Stelle dieser Art in einem Bundesland. Das Amt besteht seit November 2004 und ist durch rege Inanspruchnahme und eine große politische Unterstützung in Berlin inzwischen zu einer Institution geworden.

Bevor man die Problemfelder der Beratungsarbeit unter dem Blickwinkel der Verbraucherpolitik betrachtet, müssen weitere Aspekte ins Bewusstsein gerückt werden, die bei der Diskussion um zunehmende Eigenverantwortung und Wettbewerb wie eine Folie über die Debatte gelegt werden müssen. 


\section{These 1: Die soziale Unausgewogenheit der gesundheitlichen Versorgung wird sichtbar}

Fasst man die Anliegen, Beschwerden und Sorgen von Patienten, die sich an das Büro der Berliner Patientenbeauftragten wenden, zusammen, so kann man festhalten: Die Gesundheitsreformen belasten vor allem Menschen mit niedrigem Einkommen und führen zu einer sozial unausgewogenen Versorgung.

Die Fakten gehen schon seit Jahren durch die Presse und auch in Berlin wird deutlich, dass das Problem evident ist und eindeutig zunimmt:

- Immer mehr Menschen können sich die vielen Eigenbeteiligungen, Selbstzahlungsbeträge und Zuzahlungen nicht mehr leisten.

- Patienten verzichten auf medizinische Maßnahmen, weil ihnen das Geld für die Eigenbeiträge fehlt.

- Kassenpatienten warten auch in Berlin länger auf Termine, besonders bei Fachärzten.

- Die Wartezeiten werden ganz unverhohlen mit Einkommensargumenten der Ärzte begründet.

- Eine Sortierung nach medizinischer Notwendigkeit tritt oft in den Hintergrund, wenn Patienten schon an der

- Der Bildungsgrad entscheidet über die Wahrnehmung von Wahlmöglichkeiten. Bei der Suche nach guter Behandlung ist noch immer zuviel spezifische Kompetenz erforderlich.

- Es fehlen leicht zugängliche, patientenfreundliche und verständliche Informationen für eine Orientierung im Gesundheitswesen. Es ist auf Dauer ungeSprechstundenhilfe scheitern. recht, wenn nur bildungsstarke und gut situierte Menschen die Zugänge zu guter Versorgung kennen.

- Die regionalen Ungleichgewichte der Versorgung verschärfen sich.

Die Tatsache, dass Berlin ein einziger Versorgungsbereich für niedergelassene Ärzte ist, wirkt sich nachteilig für die Ostbezirke und für Stadtteile mit einer schwachen Einkommensstruktur aus. Das ist in den Flächenländern nicht anders (vielfach sogar noch dramatischer). Die Abwanderung von Arztsitzen insbesondere von Fachärzten in "wohlhabende « Regionen (mit mehr Privatpatienten), führt zu unzumutbaren Wartezeiten, Versorgungslücken und benachteiligt vor allem wenig mobile alte und arme Patienten. Die Möglichkeit zur Arztwahl und die Gewährleistung der Versorgung auch in Randgebieten müssen auf dem Hintergrund einer völlig überholten Bedarfsplanung mit größerem Nachdruck neu diskutiert werden.

\section{These 2: Insbesondere für alte chronisch kranke Menschen verschlechtern sich die Rahmenbedingungen}

Das Ungleichgewicht in der ambulanten fachärztlichen Versorgung trifft insDie Weigerung vieler Ärzte (auch Hausärzte) Hausbesuche zu machen, bedeutet für immer mehr alte nicht mobile Menschen eine ungerecht gesundheitliche Benachteiligung. Insbesondere alte Menschen in Pflegeeinrichtungen werden häufig nicht angemessen versorgt, wenn sie medizinische Hilfe von Fachärzten benötigen. Angehörige und Heimbeiräte nenbesondere pflegebedürftige Menschen.

nen es skandalös, wenn es nicht gelingt, für pflegebedürftige Menschen einen Facharzt anzufordern. Ein menschlich und medizinisch notwendiges Versorgungsmanagement an den Schnittstellen von Krankenhaus und Pflege funktioniert häufig schlecht. Leistungserbringer stellen $\mathrm{zu}$ oft ihre eigenen Systemlogiken über die Versorgungsnotwendigkeit. Patienten haben den Eindruck, dass ihnen teure Leistungen und Medikamente vorenthalten werden.

\section{These 3: Menschen mit Behinderung brauchen besondere Hilfe}

Die besonderen Probleme von Menschen mit Behinderung in Krankenhäusern und bei niedergelassenen Ärzten werden - auch im Büro der Patientenbeauftragten - zurzeit von Behindertenvertretern mit großem Nachdruck beschrieben und Abhilfe eingefordert. Genannt werden:

- keine ausreichenden barrierefreien Angebote in der ambulanten ärztlichen Versorgung

- bauliche Mängel in Krankenhäusern und Arztpraxen

- fehlende Unterstützung und Kostenübernahme der persönlichen Assistenz im Krankenhaus

- finanzielle Probleme bei der Abstimmung der Kostenträger

- keine Gewährleistung von Vorsorgeuntersuchungen für behinderte Menschen

- schlechte Schulung des Personals

- unzureichende Transparenzinformation über behindertengerechte Versorgung

\section{These 4: Die Beschwerden über Krankenhäuser verweisen auf strukturelle Fehlentwicklungen}

Die Patientenbeauftragte für Berlin, die Berlin School of Public Health und Gesundheit Berlin sind die Träger des neu gegründeten PatientenForums Berlin. Das PatientenForum Berlin hat sich zum Ziel gesetzt, die Patientenorientierung im Gesundheitswesen zu fördern und Patientinnen und Patienten Wissen für eine selbstbestimmte Orientierung im Gesundheitswesen zu vermitteln. Das PatientenForum Berlin stellt dazu eine Reihe von Informations-, Diskussions- und Qualifizierungsangeboten bereit, die eine aktive Beteiligung von Patientinnen und Patienten in Berlin ermöglichen.

PatientenForum, Senatsverwaltung für Gesundheit, Umwelt und Verbraucherschutz,

Die Patientenbeauftragte für Berlin, Brückenstraße 6, 10179Berlin, Telefon 030 9025-2010,

Fax030 9025-2501, E-Mail patientenbeauftragte@senguv.berlin.de, Internet

http://www.berlin.de/lb/patienten/patientenforum/index.html
Der Kostendruck und die Verdichtung der Arbeit vor allem in Krankenhäusern führen offensichtlich zur Abkehr von einem am individuellen Patienten orientierten Heilen:

- Immer mehr Patientinnen und Patienten beklagen, dass nicht sie als Person mit ihrer Krankheit im Mittelpunkt des Behandlungsgeschehens stehen, sondern die Kosten und institutionelle Vorgaben. 
- Medizinisches Personal und Pflegekräfte haben kaum mehr Zeit für Zuwendung, weil überall Personal abgebaut wurde und man merkt es Pflegekräften an, dass sie unter Stress stehen. Die Belastungssituationen des Personals können zu Fehlern und Sicherheitsmängeln führen (wie Vertreter der Gewerkschaften und der Pflegeberufe immer wieder belegen können).

- Patienten haben Sorge, dass Behandlungen aus Kostengründen nicht übernommen werden.

- Während das Thema der langen Wartezeiten bei niedergelassenen Kassenärzten in der Öffentlichkeit inzwischen bekannt ist, zeigt sich zunehmend, dass auch in Krankenhäusern gesetzlich versicherten Patientinnen und Patienten länger warten müssen als privat Versicherte.

Auffällig zugenommen haben Beschwerden über Verschiebungen von Operationsterminen und geplanten Behandlungen bei Kassenpatienten, über Infektionen mit resistenten Keimen im Krankenhaus, über Behandlungs- und Pflegefehler sowie über ein unzureichendes Versorgungsmanagement.

\section{These 5: Patienten wollen eine integrierte Versorgung}

Patienten wünschen sich eine Aufhebung der Sektorentrennung und eine echte integrierte Versorgung. Wenn sie zwischen Versorgungsbereichen, beispielsweise bei einer Krankenhausentlassung, wechseln, müssen viele ihre Behandlungsprozesse selbst organisieren und sind damit überfordert. Beschwerden beziehen sich darauf, dass

- im Krankenhaus viele Diagnoseuntersuchungen der niedergelassenen Ärzte wiederholt werden

- Krankenhäuser Behandlungen ablehnen, sich aber nicht um andere Anbieter kümmern

- nach kurzen Liegezeiten im Krankenhaus Anschlussbehandlungen nicht verbindlich geregelt sind

- sich die möglichen Kostenträger der Anschlussversorgung über die Kostenübernahme uneins sind

- die Nachsorge durch Pflegedienste nicht klappt

- sich zu wenig um die psychosoziale Weiterbetreuung gekümmert wird und
- das Angebot an spezifischen Angeboten zur integrierten Versorgung durch verschiedene Kassen zu unübersichtlich ist. Patienten wünschen sich echte integrierte Versorgung, bei der sie mit ihrem Heilungs- und Hilfeanliegen der Ausgangspunkt für die Steuerung von Versorgungsprozessen sind.

\section{These 6: Der zunehmende Wettbewerb im

Gesundheitswesen führt zu
neuer Intransparenz

Die unterschiedlichen Zwänge zur Wirtschaftlichkeit bei der gesundheitlichen Versorgung treiben Blüten, die für Patienten nicht immer von Vorteil sind. Die Ausgrenzung von Leistungen aus der gesetzlichen Krankenversicherung erlaubt es niedergelassenen Ärzten, Angebot zur Diagnose oder Behandlung wie eine Ware anzubieten und zu verkaufen. Aus den Beschwerden zur Praxis der sogenannten Individuellen Gesundheitsleistungen (IGeL) geht hervor, dass Patienten verunsichert sind oder die Angebote oft nicht den formalen Vorgaben entsprechen. Die Beschwerden beziehen sich auf:
Forderungen durch Patientenverbände für eine Veränderung gehen dahin, alle Individuellen Gesundheitsleistungen in einem Katalog zu veröffentlichen, damit Fachleute sie in einem Bewertungsverfahren kommentieren können und damit Orientierungsmöglichkeiten für Patientinnen und Patienten im Sinne einer autonomen Entscheidungsfindung möglich werden. Unbedingt notwendig ist eine offensive Beratung und Aufklärung darüber, dass solche Leistungen nicht zur Voraussetzung von akuter Behandlung gemacht werden dürfen. Patientinnen und Patienten müssen außerdem gestärkt werden, klarer auf einer transparenten Rechnungsstellung zu bestehen.

\section{These 7: Patienten brauchen Unterstützung im Vertriebs- system von Medikamenten}

Die zahlreichen Versuche der Politik, die enormen Kostensteigerungen gerade bei Arzneimitteln zu senken, haben für Patienten vielfach zu Mehrbelastungen und Überforderungen geführt. Während ein Teil der Patienten die neuen Möglichkeiten offensiv und zur eigenen Kostener-

\section{"Es fehlt ein systematisches Angebot, das Patienten das notwendige Wissen für ein souveränes Auftreten als Verbraucher zur Verfügung stellt«}

- unzureichende Aufklärung über wählbare Leistungen in der Arztpraxis

- Ausübung von Druck zur Inanspruchnahme der Angebote (z. B. Zugang zum Augenarzt erst nach Zustimmung zur Augeninnendruckmessung)

- Kosten wurden (trotz gesetzlicher Verpflichtung) nicht zuvor abgestimmt

- Unklarheiten über das Preisgefüge bei Individuellen Gesundheitsleistungen

- gezielte Verunsicherung der Patienten: Verlangen einer Unterschrift für das Ablehnen einer Leistung "auf eigene Gefahr «

- Erschleichung der Zustimmungserklärung, beispielsweise durch das Unterschieben von Formularen ohne klare Erläuterung sparnis nutzt, thematisieren Beschwerden folgende Aspekte:

- Unverständnis über Verordnungen von immer neuen Medikamenten als Folge der Rabattverträge und Festbetragsregelungen und kassenspezifischen Vorgaben für bestimmte Medikamente

- Klagen über Unverträglichkeiten bei wechselnden Generika-Verordnungen

- Unsicherheit über die Qualität und Sicherheit von Medikamenten, die im Versandhandel erhältlich sind

- und immer wieder die Klage über die zu hohen Zuzahlungen.

Deutlich wird, dass viele Patientinnen und Patienten (noch) nicht die Vorteile einiger Maßnahmen sehen können (z. B. Befreiung von Zuzahlung bei bestimmten 
vertraglichen Regelungen der Kassen mit Herstellern, die Möglichkeit über den Preis von freiverkäuflichen Medikamenten zu verhandeln). Andererseits ist das Feld so komplex, dass meist eher junge und besser gebildete Patienten hier ihre Chancen als Kunden kennen und nutzen. Es fehlen patientenverständliche Informationen und Beratungsmöglichkeiten, die das Manko der Benachteiligten ausgleichen könnten.

\section{These 8: Die Regelungen beim Zahnersatz sind für viele undurchschaubar}

Die zahnärztlichen Leistungen gehören für viele Patienten zu den kostenträchtigen und undurchschaubaren Feldern. Die Regelungen für Zahlersatz sind so komplex, aber vielfach auch so komplikationsbelastet, dass dies ein wesentliches verbraucherpolitisches Feld ist. Das hat damit zu tun, dass Zahnbehandlungen häufig seelisch stark besetzt sind, vor allem aber auch damit, dass die zahnärztliche Versorgung und die Erstellung von Zahnersatz eine Sonderrolle im Gesundheitswesen einnehmen. Hier überschneiden sich Leistungsregelungen aus der gesetzlichen Versicherung und der Pflicht zu privaten Kostenübernahme mit einem komplexen (auch internationalen) Anbietermarkt. Für Patienten als Verbraucher wird es meist dann problematisch, wenn es zu Problemen bei Gewährleitungsrechten nach offensichtlichen oder vermuteten Behandlungsfehlern kommt. Beschwerden beziehen sich daher auf Probleme mit Kostenplänen, fehlende Vergleichsinformationen, Sorgen vor zu hohen Kosten, unzulänglicher Beratung durch die Krankenkassen sowie unzulänglicher Markttransparenz. Auch hier fehlt das notwendige Wissen bei den Patienten, wer wie helfen kann und faktisch hilft.

\section{These 9: Fehlende Qualitätstransparenz behindert souveränes Verbraucherverhalten}

$\mathrm{Zu}$ den häufig gestellten Fragen in Patientenberatungsstellen gehört die Suche nach »Spezialisten « im Gesundheitswesen. Dahinter verbirgt sich das
Problem, dass Patienten bei der Wahl von Ärzten oder Krankenhausleistungen eigentlich kaum Informationen über die Güte oder Qualität der Leistungserbringer in ihnen zugänglichen Medien und in verständlicher Form zur Verfügung stehen.

Es hat zwar in den letzten Jahren - insbesondere für Krankenhäuser durch die gesetzliche Verpflichtung zu Qualitätsberichten und zur Veröffentlichung von Ergebnissen einer vergleichenden Qualitätssicherung - erhebliche Fortschritte gegeben. Aber das Wissen ist bei weitem noch nicht verständlich genug aufbereitet und bezieht sich bisher nur auf höchstens ein Fünftel der Leistungen.

Wenn die Politik will, dass Patienten sich wie souveräne Akteure im Markt an Entscheidungen beteiligen und Leitungen selber wählen, ist hier noch erheblich mehr Aufwand für Transparenz und Verständlichkeit notwendig.

\section{These 10. Die Stärkung der Souveränität von Patienten verlangt Information und Beratung}

Bei all den Themen wird immer wieder deutlich, dass es an Information, Beratung, Aufklärung und Unterstützung für Patienten, vor allem aber an Markttransparenz fehlt. Damit ist nicht die Tatsache gemeint, dass es zu wenige unabhängige und neutrale Patientenberatungsstellen gibt. Durch Finanzierung der Krankenkassen wurden hier in den letzten Jahren bei Verbraucherzentralen und Patientenverbänden zusätzliche Angebote geschaffen, die zwar noch nicht ausreichen, aber immerhin ein Fortschritt sind. Dringlich sind vielmehr ein schlüssiges Konzept und ein systematisches Angebot, das Patienten das notwendige Wissen und vor allem die erforderliche Kompetenz für ein souveränes Auftreten und den Wechsel in der eigenen Rollenwahrnehmung zur Verfügung stellt.

Neben Wissen über formale Rechte, über Grundlagen zu Gesundheitsfragen und Behandlungsalternativen geht es nämlich vor allem darum, als Akteur in einem komplexen System mutiger und eigenständiger aufzutreten. Natürlich ist eine Person mit Schmerzen, mit der Angst vor einer schweren gesundheitlichen Ein- schränkung, mit dem Bedürfnis, sich vorbehaltlos einer kompetenten Fachperson anvertrauen zu können, nicht vergleichbar mit der Käuferin eines Autos oder einer Handwerkerleistung. Dennoch muss diese Mischung in der Vermittlung aus Wissen und Kompetenz im Auftreten als neues Angebot entwickelt werden, damit Patientinnen und Patienten sich aktiver als Verbraucher im Gesundheitsmarkt bewegen können und damit sie zu einem Partner werden, mit dem die Leistungsanbieter rechnen (müssen) und den sie ernst nehmen. Vorschläge in diese Richtung gehen dahin, das Thema zu Gegenstand schulischer Bildungspläne zu machen, andere haben Konzepte für eine $\mathrm{Pa}$ tientenuniversität (z. B. in Hannover) erprobt.

In Berlin wird mit dem »PatientenForum « (vgl. Kasten Seite 104) eine Plattform geschaffen, die kontinuierlich Wissen vermitteln will und Diskussionsangebote mit Experten macht, die öffentlich so begleitet ist, dass zunehmend mehr Individuen die Möglichkeit zur eigenen Schulung für die Rolle als aktive Patienten nutzen.

\section{Resümee}

Information, Beratung und Schulung werden den notwendigen Rollenwechsel vom hilflosen Patienten zum mündigen Verbraucher allein nicht bewältigen. Erforderlich ist zudem, die Verbraucherrechte der Patienten zu stärken. So ist das Vorhaben der Beauftragten des Bundes für die Belange der Patienten zu begrüßen, eine gesetzliche Weiterentwicklung hin zu Vertragsrechten für Patienten voranzutreiben.

Ebenso wichtig ist, dass Patienten in möglichst vielen Feldern kollektive Beteiligungsrechte eingeräumt werden, wie sie schon für den Gemeinsamen Bundesausschuss (zur Festlegung von Leistungen der gesetzlichen Krankenversicherung) geschaffen wurden ( $\mathbb{S} 104$ f. SGB V). Denn überall dort, wo Patienten ihre Erfahrungen und ihre Erwartungen einbringen können, kann die Sichtweise der eigentlich »Betroffenen « in die Planungen, Regelungen und förmlichen Vorgaben berücksichtigt werden. Patientenorientierte Verbraucherpolitik braucht eine offensive Beteiligungskultur der $\mathrm{Pa}$ tienten. 